\title{
Longitudinal quadrupole instability and control in the Frascati DAФNE electron ring
}

\author{
A. Drago, A. Gallo, A. Ghigo, and M. Zobov \\ INFN-LNF, P.O. Box 13, I-00044 Frascati (RM), Italy \\ J. D. Fox and D. Teytelman \\ Stanford Linear Accelerator Center, 2575 Sand Hill Road, Menlo Park, California 94025, USA \\ (Received 14 January 2003; published 7 May 2003)

\begin{abstract}
A longitudinal quadrupole ( $q$-pole) instability was limiting the maximum stable current in the DAФNE $e^{-}$ring at a level of $\sim 700-800 \mathrm{~mA}$. In order to investigate the phenomenon, the instability threshold has been measured as a function of various machine parameters as radio frequency voltage $\left(V_{\mathrm{rf}}\right)$, momentum compaction $\left(\alpha_{c}\right)$, number of bunches, fill pattern, etc. An unexpected interaction with the longitudinal feedback system, built to control the dipole motion, has been found and a proper feedback tuning has allowed increasing the threshold. The maximum stable beam current has now exceeded $1.80 \mathrm{~A}$ and it is no longer limited by the quadrupole instability.
\end{abstract}

DOI: 10.1103/PhysRevSTAB.6.052801

PACS numbers: 29.27.Bd

\section{INTRODUCTION}

DAФNE is a $\Phi$-Factory, $e^{+} / e^{-}$collider in operation at the Laboratori Nazionali di Frascati, INFN, Italy, for physics experiments since 1999, with gradually increasing peak and integrated luminosities [1]. In order to reach the high luminosity required, in the $10^{32} \mathrm{~cm}^{-2} \mathrm{~s}^{-1}$ range, multibunch beams with currents higher than $1 \mathrm{~A}$ must be stored in both rings of the collider. The design current per single bunch of $44 \mathrm{~mA}$ has been successfully exceeded in both rings. In Table I some relevant DAФNE parameters are summarized.

In multibunch operations, a longitudinal quadrupole ( $q$-pole) instability was limiting the maximum stable current in the DAФNE $e^{-}$ring at a level of $\sim 700-800 \mathrm{~mA}$. The experimental study of the instability has allowed finding measures to damp or avoid it and storing a stable $e^{-}$beam with more than $1.80 \mathrm{~A}$. Below, the longitudinal feedback system is described in Sec. II, the quadrupole instability phenomenology and its threshold in dependence by different machine parameters are presented in Sec. III, a cure to damp the instability is described in Sec. IV, a discussion on the phenomenon is outlined in Sec. V, and the conclusions are summarized in Sec. VI.

\section{THE LONGITUDINAL FEEDBACK SYSTEM}

Considering the longitudinal dynamics in DA $\Phi N E$ main rings, strong coupled-bunch synchrotron (dipole) oscillations make active damping systems necessary. In order to cope with the instability, broadband

TABLE I. Machine parameters.

\begin{tabular}{lccc}
\hline \hline \multicolumn{1}{c}{ Parameter description } & Value & Unit & Notes \\
\hline Main rings (MR) & 2 & & \\
Interaction points (IP) & 2 & & (Kloe in IP1, Dear in IP2) \\
Single beam energy & 510 & $\mathrm{MeV}$ & \\
Center of mass energy & 1.02 & $\mathrm{GeV}$ & \\
Revolution frequency $\left(1 / T_{0}\right)$ & 3.069 & $\mathrm{MHz}$ & \\
Radio frequency (rf) cavities & 1 & & (for each ring) \\
rf frequency & 368.26 & $\mathrm{MHz}$ & \\
rf voltage $\left(V_{\text {rf }}\right)$ & $80-200$ & $\mathrm{kV}$ & (range) \\
rf voltage $\left(V_{\mathrm{rf}}\right)$ & 120 & $\mathrm{kV}$ & (typical) \\
Synchrotron frequency & 30 & $\mathrm{kHz}$ & (typical) \\
Longitudinal radiation damping & 17.8 & $\mathrm{~ms}$ & (typical) \\
Momentum compaction $\left(\alpha_{c}\right)$ & 0.021 & & (electron ring) \\
Betatron tunes $\left(\nu_{x} / \nu_{y}\right)$ & $5.11 / 5.15$ & & \\
Harmonic number & 120 & & (design value) \\
Max number of bunches & 120 & & (achieved 200 mA) \\
Particles per bunch & $9.0 \times 10^{10}$ & & $\mathrm{~mA}$ \\
Max nominal single bunch current & 44 & & \\
\hline \hline
\end{tabular}


bunch-by-bunch longitudinal feedbacks (LFB) were installed in each ring operating since 1998. The systems have been developed in collaboration with PEP-II/SLAC and ALS/Berkeley [2-9]. The $n$th bunch can be described as an individual harmonic oscillator moving rigidly in the longitudinal plane (energy oscillations) [10,11], according to the equation:

$$
\ddot{\tau}_{n}+2 d_{r} \dot{\tau}_{n}+\omega_{s}^{2} \tau_{n}=-\frac{\alpha_{c} e}{E_{0} T_{0}} V_{n}^{\mathrm{wk}}(t)
$$

where $\tau_{n}$ is the arrival time (time delay) of the $n$th bunch relative to the synchronous particle, $d_{r}$ is the natural radiation damping, $\omega_{s}$ is the natural (synchrotron) oscillation frequency, $\alpha_{c}$ is the momentum compaction, $E_{0}$ is the nominal energy, and $e V_{n}^{\mathrm{wk}}(t) / T_{0}$ is the rate of energy loss due to the superposition of the wake forces of the other bunches.

The action of the feedback consists of individual kicks to each bunch increasing the damping term $d_{r}$. In the presence of an active feedback Eq. (1) becomes

$$
\ddot{\tau}_{n}+2 d_{r} \dot{\tau}_{n}+\omega_{s}^{2} \tau_{n}=\frac{\alpha_{c} e}{E_{0} T_{0}}\left[V_{n}^{\mathrm{fb}}(t)-V_{n}^{\mathrm{wk}}(t)\right]
$$

where $V_{n}^{\mathrm{fb}}(t)$ is the feedback kick given to the bunch $n$ th.

In Fig. 1 a block diagram of the system is shown: for each bunch a longitudinal phase signal (error signal) is acquired by the LFB that provides a correction kick at the passage of the bunch through the kicker. The main functions requested to a feedback system are the following: (i) detect the bunch longitudinal oscillations; (ii) provide adequate feedback loop gain at the selected frequency range; (iii) provide a $\pi / 2$ phase shift at the oscillation frequency.

The LFB system consists of three main blocks:

(i) An analog front end followed by a programmable delay, to detect the error signal of each bunch. The function of the programmable delay is to synchronize the output signal of this block with the digital part. (ii) A digital part, to manage separately the signal of every bunch with individual passband filters having a convenient gain and phase response. The global phase response of the feedback must give a $\pi / 2$ phase shift at the dipole frequency.

(iii) An analog back end (BE) followed by a second programmable delay, power amplifiers, and cavity kicker. The BE programmable delay has the task of synchronizing the peak of the $n$th kick with the passage of the $n$th bunch through the kicker.

The LFB system is broadband, in time domain and on a bunch-by-bunch basis. This means that it manages the error signal of each bunch independently from the other bunch signal. In Fig. 2 a scheme of the front-end block is shown: it acts as a phase $(\phi)$ detector, with $\phi=\omega_{\mathrm{rf}} \tau$. A sum signal from a four button monitor is sent to a comb generator that outputs a tone burst at $6 \mathrm{rf}$. This is sent to a mixer followed by a low pass filter producing an output voltage proportional to the phase difference between the bunch input signal and the reference from the master oscillator. This FE_signal_out goes to the following LFB section, while a signal copy (FE_monitor_out) goes to an oscilloscope to be monitored for diagnostic purposes.

In the digital part (Fig. 3), the FE_signal_out is sampled by an A/D converter at $r f$ frequency, and then demultiplexed to separate the signal of each bunch.

A digital signal processor (DSP) farm is used to implement a passband filter [finite impulse response (FIR) or infinite impulse response (IIR) [12]]. The number of taps, gain with sign, center frequency, filter shape, and phase response are programmable by the users, but the loaded filters have to be equal for all the bunches, even if it is possible to run an "exception" filter for just one bunch. This limit depends upon the implemented system architecture (hardware and software). Amplitude and phase response of a FIR filter implemented for high current electron beam are shown in Fig. 4. The output value computed for each bunch is put in a hold buffer memory

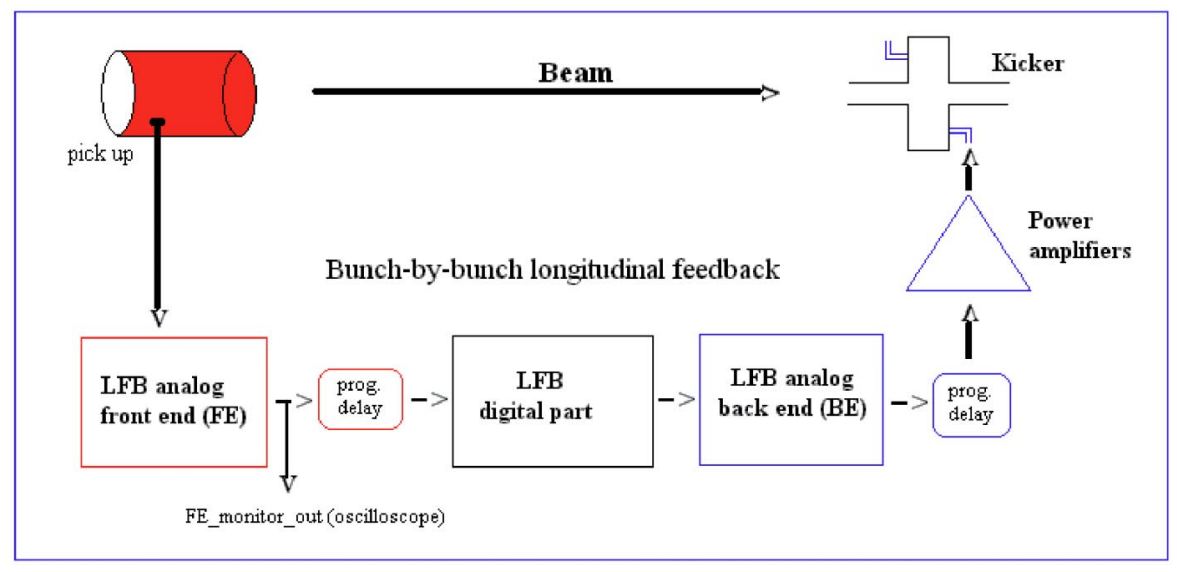

FIG. 1. (Color) Block diagram of the longitudinal feedback system. 


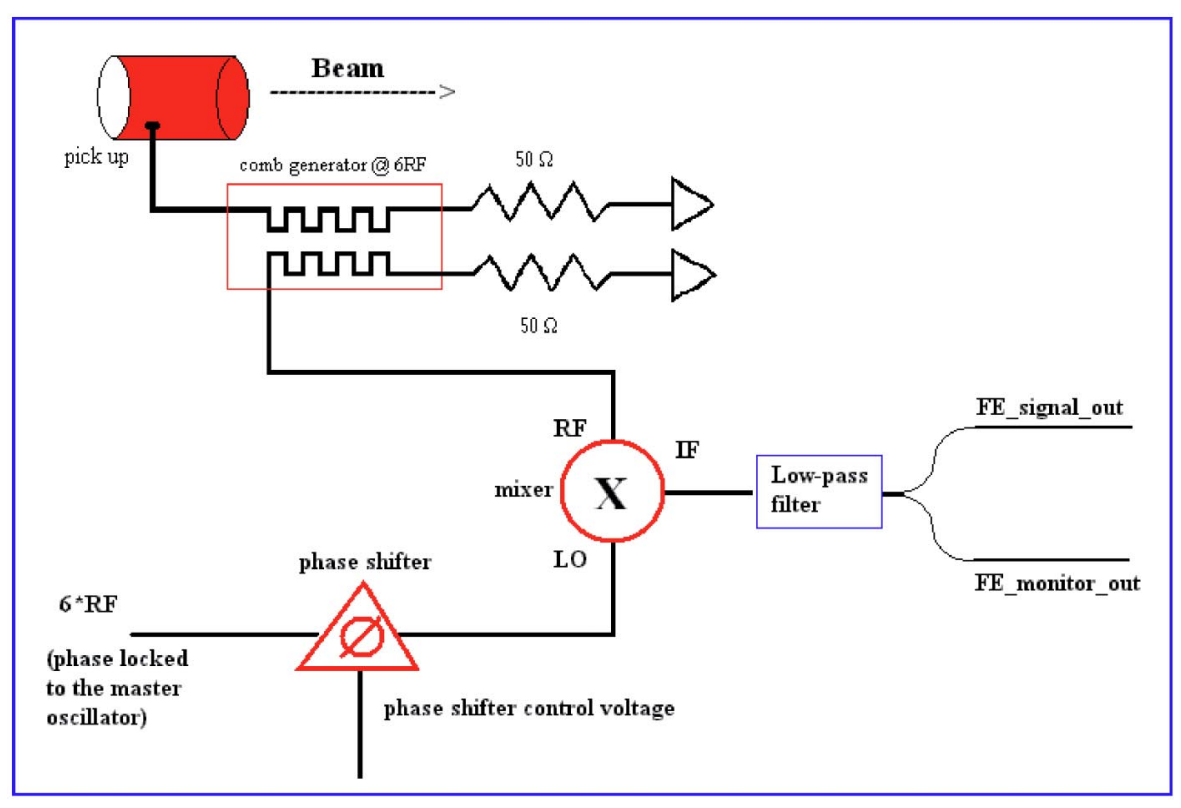

FIG. 2. (Color) Block diagram of the longitudinal feedback front end.

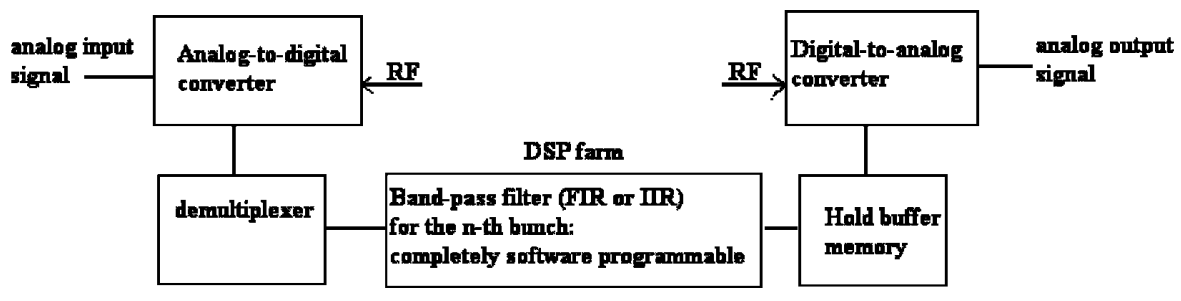

FIG. 3. Block diagram of the LFB digital part.

together with the values of the other bunches; the memory is continuously scanned for a digital-to-analog converter (DAC) producing the analog correction signal.

In the back-end block, the analog signal coming from the DAC modulates in amplitude a quadrature phase shift keying (QPSK) modulated carrier centered at 13/4 $\mathrm{rf}$ which is locked in phase with the master oscillator; see Fig. 5. After this block, a programmable delay line provides the correct synchronization with the bunch passage and three $250 \mathrm{~W}$ power amplifiers feed a cavity kicker centered at $13 / 4 \mathrm{rf}$ center frequency $[13,14]$. To find the best value for the BE delay, the method used consists in forcing one bunch to oscillate longitudinally and measuring the amplitude of the bunch oscillations versus delay values. A LFB software feature, using the system in open loop allows generating the excitation signal by a program in the DSP farm. The operator chooses the sinusoid frequency in the range of the synchrotron one. Figure 6 shows the longitudinal back-end response to the excitation signal as a function of the back-end delay value. To obtain the measurement, the pickup phase signal (FE_monitor_out) is sent to a spectrum analyzer to monitor the bunch oscillations excited by the system. The response top value of the spectrum (for each delay value) is recorded to form a point of the pattern. The periodic behavior is due to the kicker periodicity and it is equal to the half period of the kicker oscillations (13/4 rf). This method is used to choose the best BE programmable delay value for damping the dipole oscillations. The bunch passage must be synchronized with the center of the highest lobe to exploit most of the power. The useful period is $418 \mathrm{ps}$ and, when the LFB works in closed loop, contiguous lobes have opposite phases.

Figure 7 shows an example of the frequency power spectrum of an unstable beam (LFB off). This figure and the following similar ones are obtained using a powerful real time spectrum analyzer, the HewlettPackard 3587s. The highest power spectrum line (with the square marker) corresponds to a revolution harmonic (the 117th in our case). The nearby synchrotron sidebands with the height modulated by the $J_{0}$ Bessel function reveal strong dipole oscillations (in this figure and in the following only the sidebands on the right are entirely shown). The injected pattern is a train of 45 bunches, each one spaced by one empty bucket, and a $25 \%$ gap for ion 

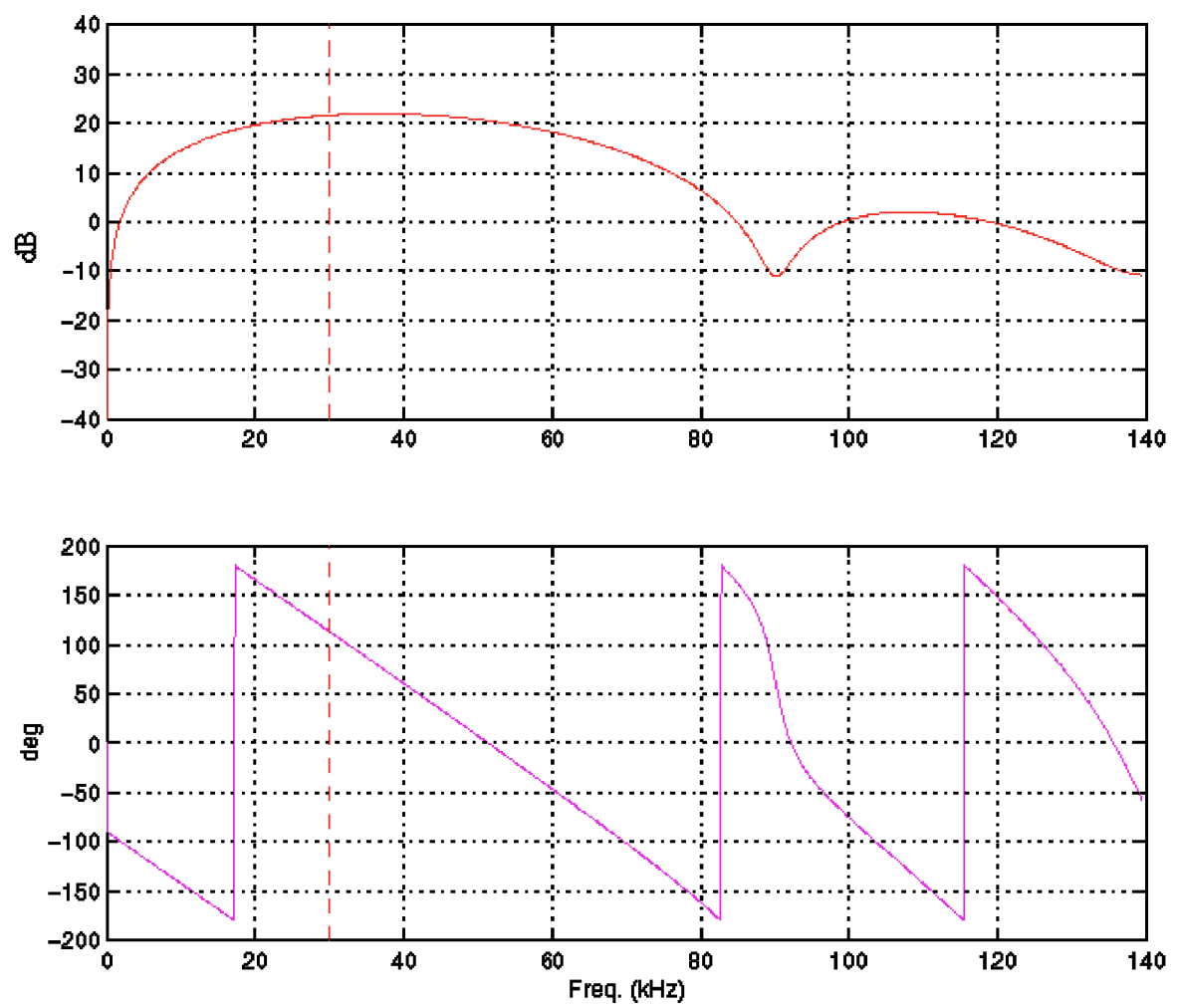

FIG. 4. (Color) Amplitude and phase response versus frequency of a FIR filter implemented in the DSP farm for high current electron beams. The synchrotron frequency is shown with the dotted line.

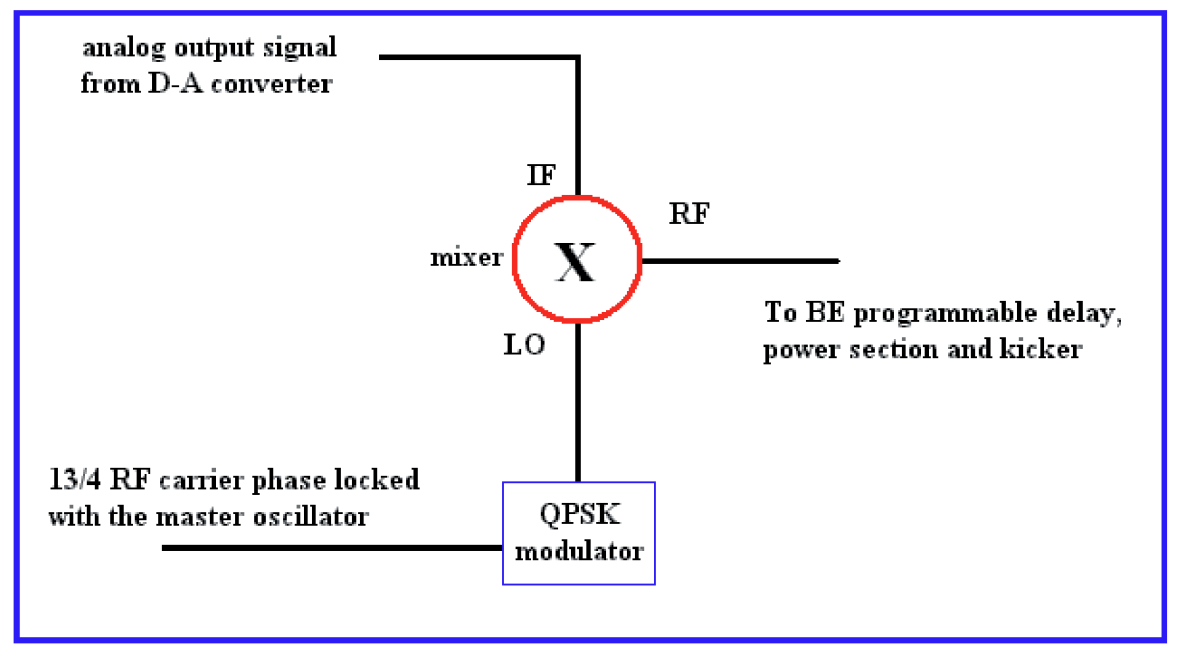

FIG. 5. (Color) LFB back-end block diagram.

cleaning follows the train. The total beam current is $\sim 300 \mathrm{~mA}$ and $V_{\mathrm{rf}}=120 \mathrm{kV}$.

In the same situation, turning on the longitudinal feedback, no sidebands are around the revolution harmonic; see Fig. 8.

\section{QUADRUPOLE INSTABILITY}

These LFB systems work fairly well, but, during all of 2002 and sometimes in the previous years, an unexpected longitudinal quadrupole instability was limiting the total current to $\sim 800 \mathrm{~mA}$ in the $e^{-}$ring. This instability appeared usually above $600 \mathrm{~mA}$, producing harmful effects for the beam-beam interaction and also limiting the maximum stored current.

\section{A. Phenomenon description}

To introduce the argument, let us consider, as an example, the same usual case of $45 e^{-}$bunch configurations 


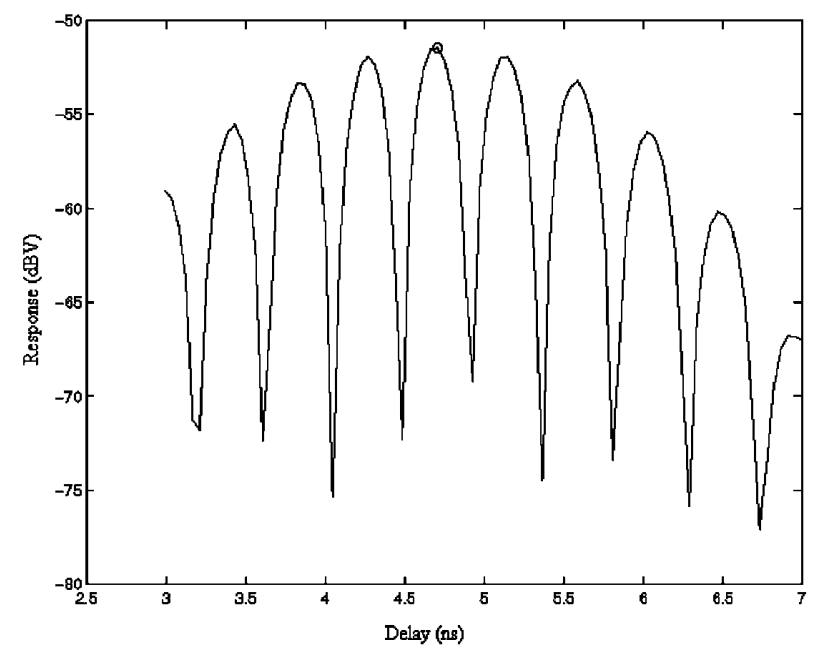

FIG. 6. Longitudinal back-end response (dBV) versus BE programmable delay (ns).

stored in the odd buckets, as the pattern considered for Figs. 7 and 8.

At high currents (between 600 and $800 \mathrm{~mA}$ ), with the previous injected pattern or any other multibunch fill pattern, with LFB on, in the electron main ring a quadrupole line (without the dipole one) appeared in the beam spectrum as indicated in Fig. 9 by the marker, limiting further current injections. The current limit consists of the fact that new injections can produce a loss of bunches and/or a loss of LFB control with a successive large decrease of the total beam current.

Another aspect should be considered: in the case shown in Fig. 9, it is possible to observe that the $q$-pole frequency is at $58.75 \mathrm{kHz}$, while the second harmonic of the synchrotron frequency is at $60 \mathrm{kHz}$, with a difference of $-1.25 \mathrm{kHz}$ from the zero current line.

\section{B. Relevant machine parameters}

In order to overcome the current limit, the $q$-pole instability threshold has been measured as a function of the following machine parameters: radio frequency voltage $\left(V_{\mathrm{rf}}\right)$, momentum compaction $\left(\alpha_{c}\right)$, orbit bumps (considering a trapped mode), injected patterns and number of bunches, bunch length, and LFB back-end setup.

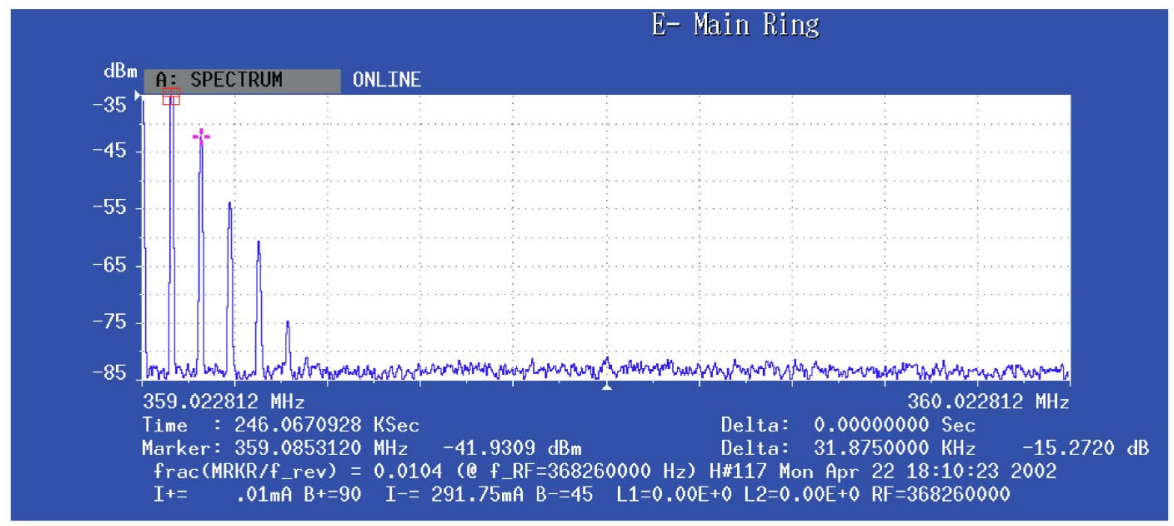

FIG. 7. (Color) Multibunch beam power spectrum at $\sim 300 \mathrm{~mA}$ with LFB off. The square marker is on the revolution harmonic; the cross marker is on the dipole oscillation right sideband.

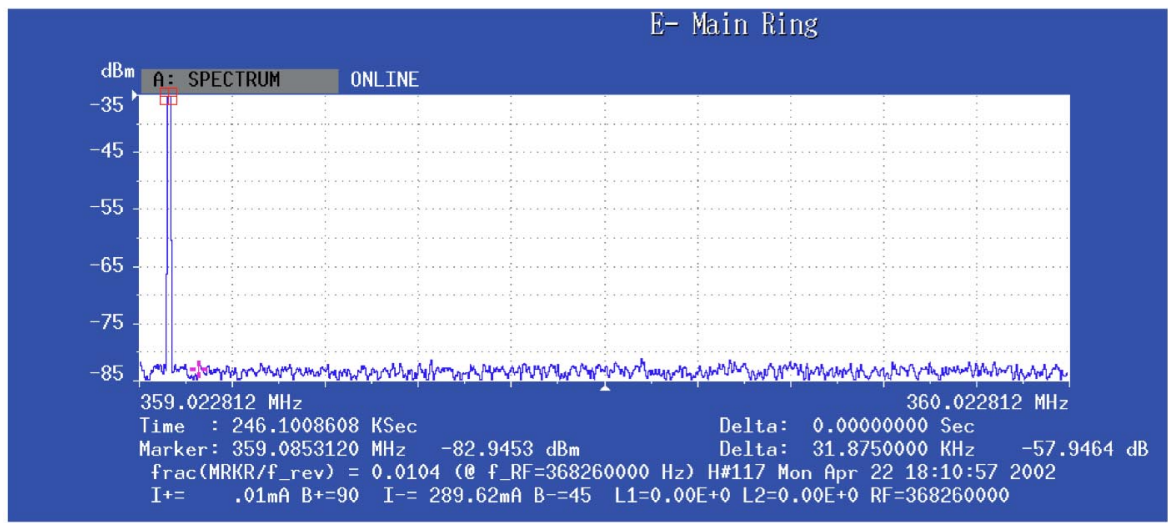

FIG. 8. (Color) Multibunch beam power spectrum at $\sim 300 \mathrm{~mA}$ with LFB on. The square marker is on the revolution harmonic. 


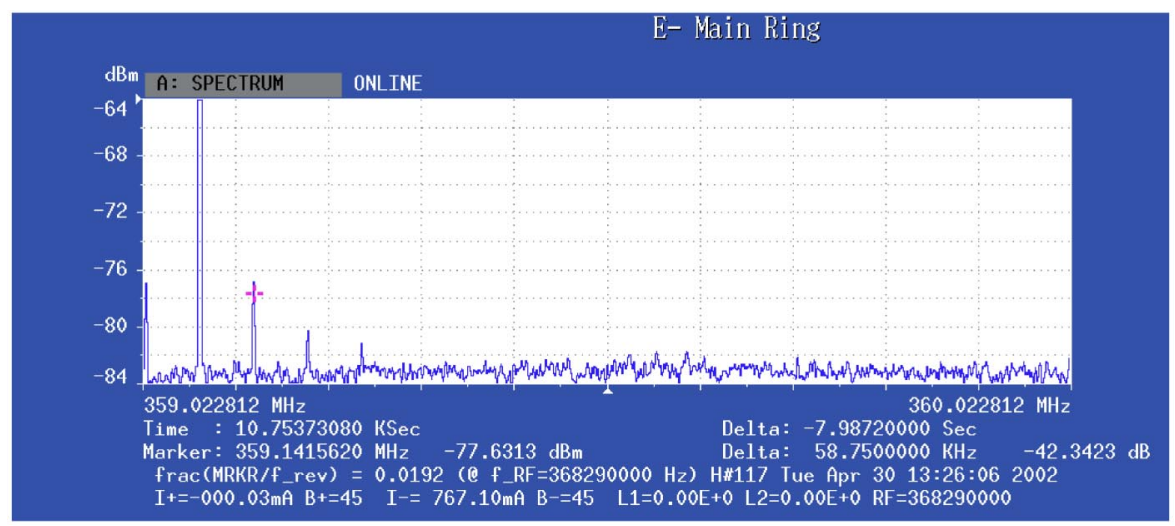

FIG. 9. (Color) Multibunch beam power spectrum showing quadrupole instability (the right sideband is indicated by the cross marker). The beam current is $\sim 760 \mathrm{~mA}, V_{\mathrm{rf}}$ is $120 \mathrm{kV}$, and LFB is on.

\section{First measurements}

A clear variation of the $q$-pole threshold was observed as a function of the rf voltage: injecting a 47/60 bunch pattern, the threshold has been measured at $\sim 550 \mathrm{~mA}$ with $V_{\text {rf }}=120 \mathrm{kV}$ and at $\sim 750 \mathrm{~mA}$ with $V_{\mathrm{rf}}=170 \mathrm{kV}$. For the intermediate voltages, we have found proportional thresholds (this kind of measure is not very precise).

The dependence on momentum compaction has been evaluated. A $\sim 10 \%$ increase of the $\alpha_{c}$ value (from 0.03 to 0.033 ) has allowed us to increase the quadrupole threshold by $\sim 17 \%$ (from $\sim 750$ to $\sim 880 \mathrm{~mA}$ in 47 bunches) (October 2001). However, variations of this parameter have not given a definitive solution for the instability damping.

Afterwards the $q$-pole threshold has been measured varying the number of bunches and injected patterns. It has been found that the threshold increases with the number of bunches, but this is neither conclusive nor sufficient to cancel the current limit.

\section{Two different behaviors}

The measurement that has indicated more clearly the terms of the problem was that of a single bunch current $q$-pole threshold versus rf voltage, with LFB off and on; see Fig. 10.

Comparison shows that the lowest threshold with LFB on corresponds to no $q$-pole evidence with LFB off. In general, the two situations (with and without LFB) have different behavior as if they were two different types of quadrupole instabilities at all. This persuasion has led us to study any possible interaction between LFB and $q$-pole instability threshold.

\section{E. Bunch length and LFB back end}

Figure 5 has shown the single bunch longitudinal backend response as a function of the back-end delay in the cavity kicker of the LFB system. The useful period is $<418$ ps and it is followed by an inversion of the feedback

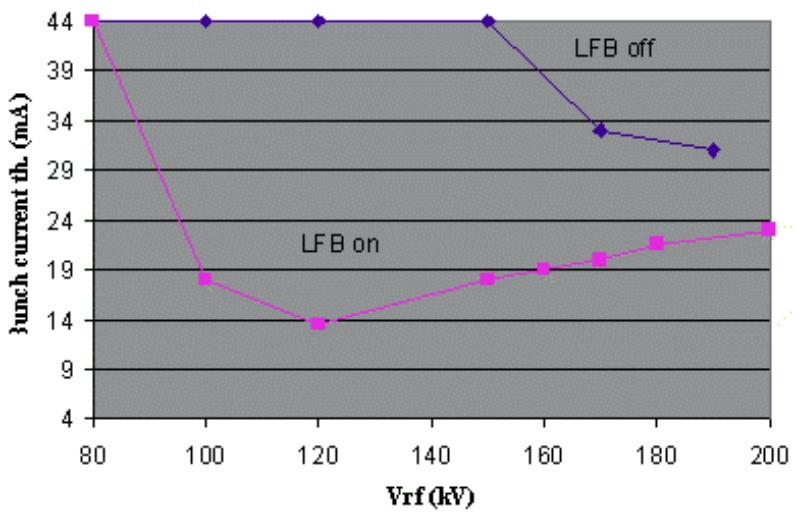

FIG. 10. (Color) Single bunch $q$-pole threshold (09/13/2001, note: $44 \mathrm{~mA}=$ no threshold).

phase. On the other hand, the measured $e^{-}$bunch length (FWHM) is $<144$ ps at $1 \mathrm{~mA}$, it is $\sim 220 \mathrm{ps}$ at $15 \mathrm{~mA}$, and it grows up to 300 ps at $39 \mathrm{~mA}$, with $V_{\text {rf }}$ equal to 120 kV [15] (see Fig. 11).

Considering the data in Secs. III D and III E, we have investigated whether a bunch length comparable with (half $\mathrm{BE}$ period) could drive a cross talk between LFB and $q$-pole instability.

\section{THE INSTABILITY CURE}

Measuring the $q$-pole threshold versus LFB back-end delay, we have experimentally found that increasing conveniently the $\mathrm{BE}$ delay timing (i.e., kicking the bunch tail) produces higher thresholds or cancels the instability and decreasing delay (i.e., kicking the bunch head) lowers $q$-pole threshold [16]. A plot is drawn in Fig. 12. Looking at this picture, and considering a bunch modeled as two macroparticles (head and tail) [17], the $150 \mathrm{ps}$ delay makes it possible to kick at zero power (no kick) the bunch head and full power the bunch tail. This is true if the distance between the peak and zero (209 ps) is comparable to the bunch length ( $220 \mathrm{ps}$ at $15 \mathrm{~mA}$ ). 


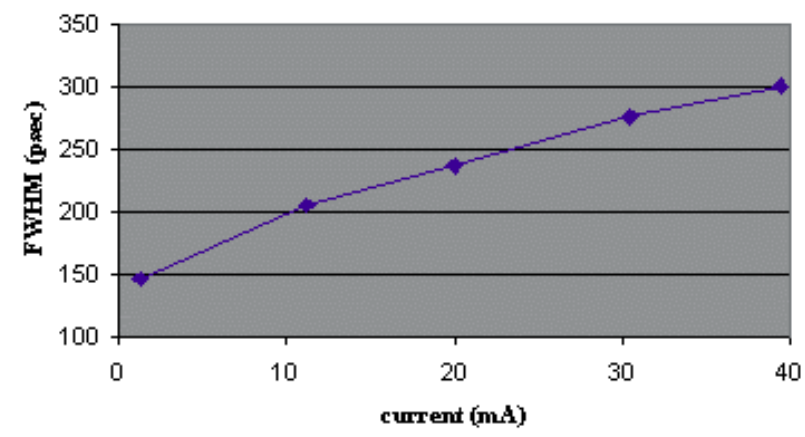

FIG. 11. (Color) Bunch length versus single bunch current: $e^{-}$ bunch length (FWHM) @ $V_{\mathrm{rf}}=120 \mathrm{kV}(11 / 14 / 2001)$.

After this discovery, one of the authors has increased by $150 \mathrm{ps}$ the LFB back-end delay with respect to the dipole correct timing. In this way the responses to the dipole and to the quadrupole motions are well balanced at high current; it has been possible to avoid $q$-pole instability for all typical collision cases and to store more than $1800 \mathrm{~mA}$ of stable electron beam in April 2002 (see Fig. 13).

Using the LFB kick delay, we discovered that a suitable phase offset in the front-end signal is always necessary. In Fig. 14 a real case with the two front-end LFB synchronous phase signals (FE_monitor_out) is shown by an oscilloscope: in channel 1 the positron beam signal (black), in channel 2 the electron beam signal (green). They are obtained comparing the bunch-per-bunch synchronous phase with a reference as shown in Fig. 2. To maintain the two beams stable, it is necessary to have a phase offset different from zero and of the same sign for all the bunches of each beam. More precisely, in the case shown in Fig. 14, the $e^{+}$FE phase signal has positive offset for the bunch while the $e^{-}$phase signal has negative offset. In the case shown the stored beams (100 bunch train followed by a 20 bucket gap) are colliding. The currents are $\sim 1 \mathrm{~A}$ in each ring.

Considering these results, a counterproof was performed: turning on the LFB, injecting a single bunch with $V_{\mathrm{rf}}=120 \mathrm{kV}$, and current $>26 \mathrm{~mA}$, and decreasing by 150 ps from the peak the LFB back-end delay, a $q$-pole motion has been excited (note that this happens also in the $e^{+}$rings at higher currents), as shown in Fig. 15. Again, the shift of the LFB front-end phase influences the phenomenon, because only with a negative offset in the frontend phase is it possible to excite the quadrupole motion.

\section{DEVELOPMENTS AND DISCUSSION}

The cure, found experimentally, is very reliable, but to understand the underlying mechanism, other tests have been done to study more deeply the phenomenon.

First of all, during the summer of 2002, with the aim of increasing the DAФNE luminosity, we have applied a LFB setup to damp both dipole and quadrupole to the other main ring $\left(e^{+}\right)$, observing beneficial consequences: a more stable and flatter beam at very high current and in collision. This test has demonstrated that the $q$-pole instability is present in both main rings with similar behavior but different thresholds. In fact in Fig. 16 the positron beam frequency power spectrum shows a large quadrupole oscillation before to be damped delaying the back-end LFB kick. In this figure, different from the other ones obtained by the HP3587s spectrum analyzer,

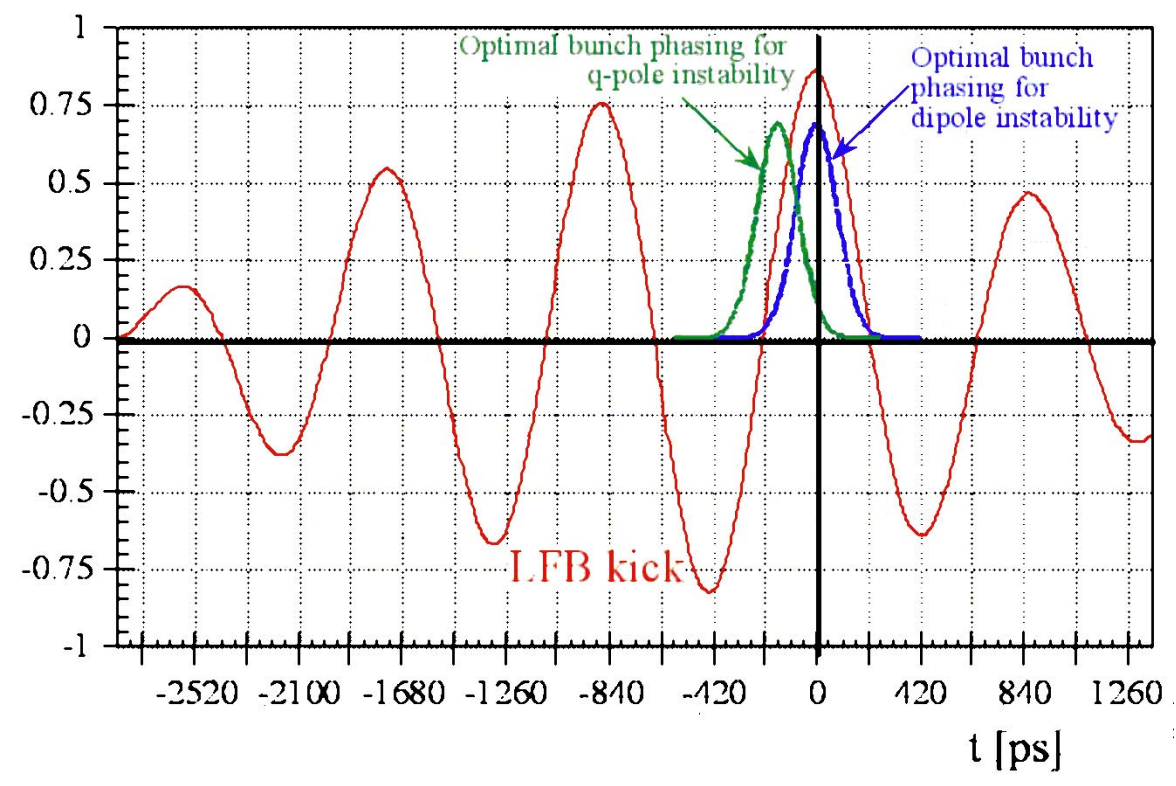

FIG. 12. (Color) BE timing versus bunch passage in the kicker. In red: the LFB kick; in blue: the bunch with optimal phase for the dipole instability; in green: the bunch with optimal phase for $q$-pole and dipole instability (note: the beam is seen traveling from right to left). 


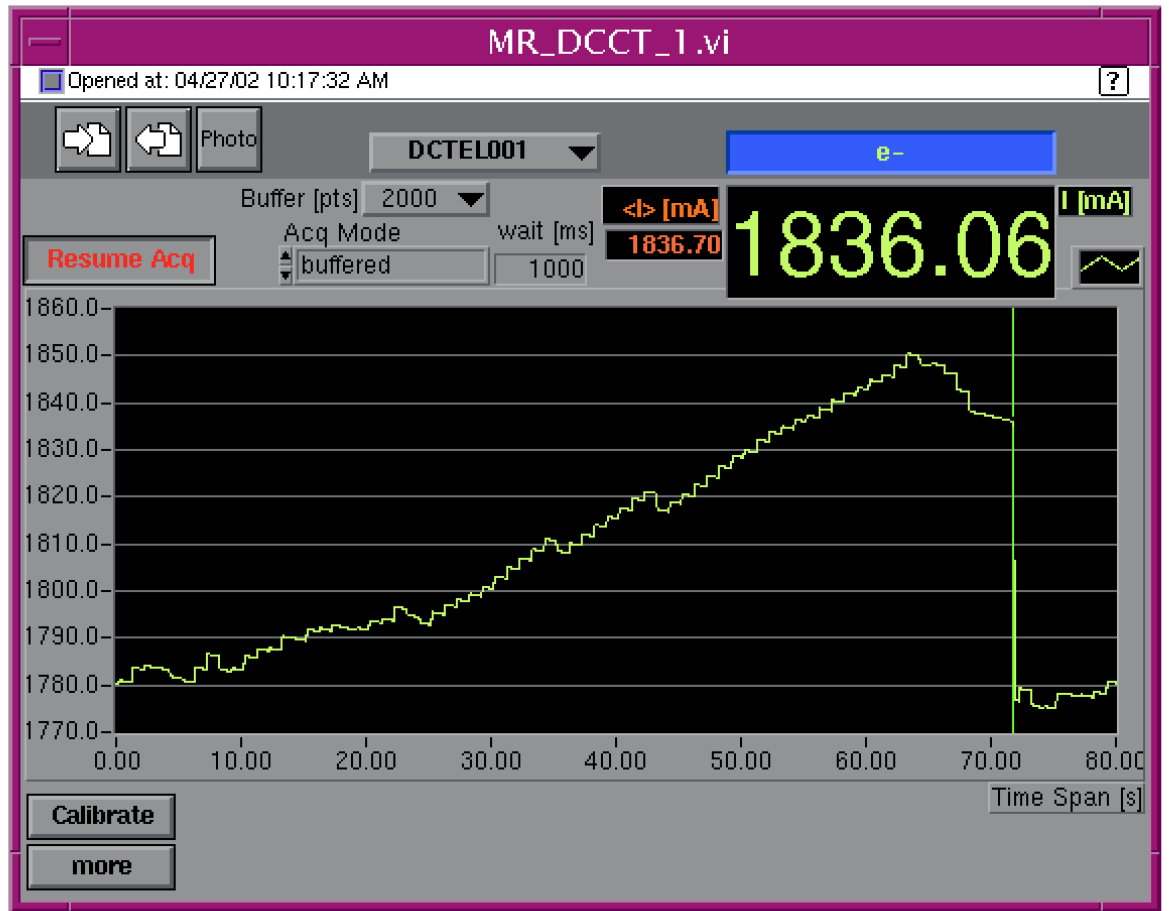

FIG. 13. (Color) The control system window showing the maximum current achieved with a stable $e^{-}$beam (1850 mA, 90 bunches, April 2002).

the highest peak (the 76th revolution harmonic) is in the center and the span is smaller. The quadrupole motion shows only the peak on the left side, indicating a strong quadrupole mode No. 76. The machine was set to collide in IP2 for the second experiment (DEAR) with a train of
100 contiguous bunches; the currents were $I^{+}=874 \mathrm{~mA}$ and $I^{-}=1027 \mathrm{~mA}$.

Then, in November 2002, we have implemented in the DSP farm a very narrow bandpass filter, i.e., a notch in the quadrupole frequencies (see Fig. 17): we have used it

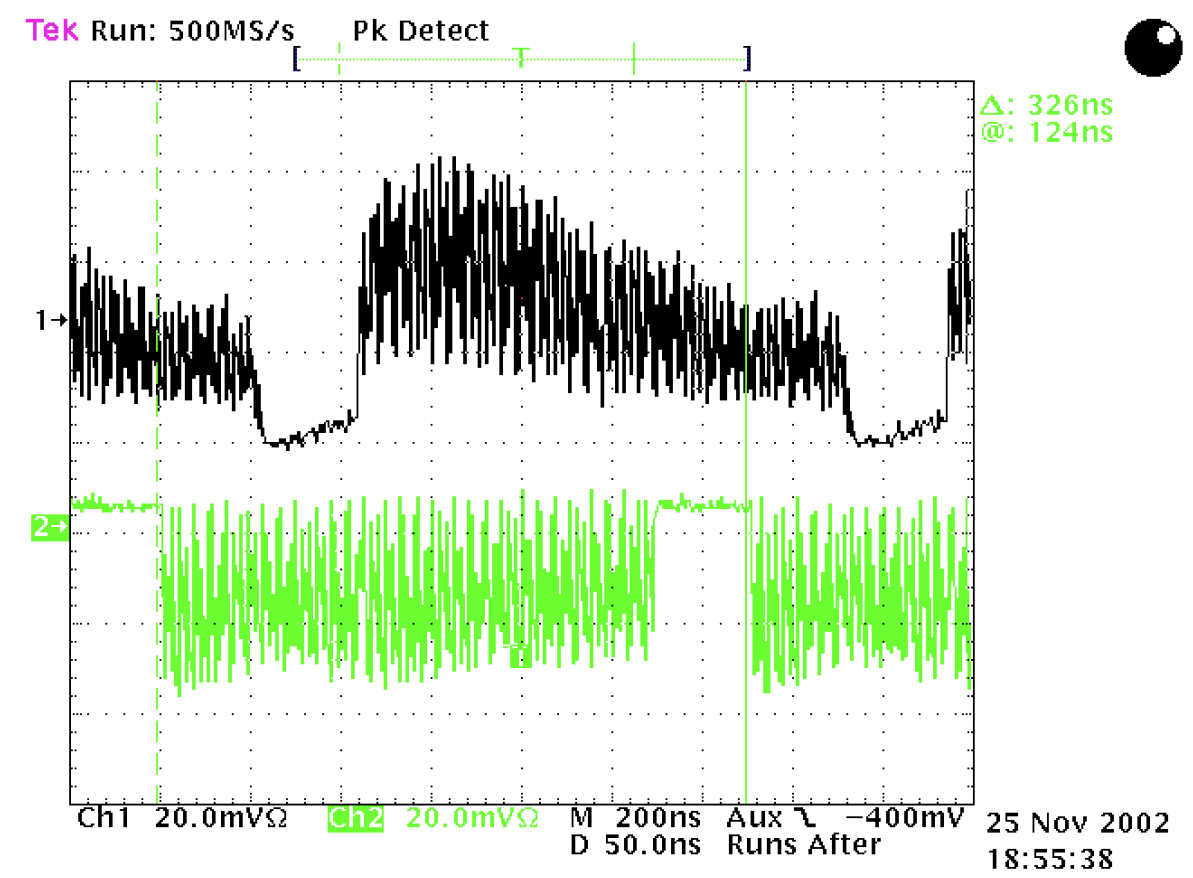

FIG. 14. (Color) LFB front-end output signals FE_monitor_out (synchronous phase monitor) shown by a Tektronix TDS 700 oscilloscope: in channel 1 (black) the positron beam, in channel 2 (green) the electron beam, both with 100 bunches colliding and currents $=\sim 1$ A. 


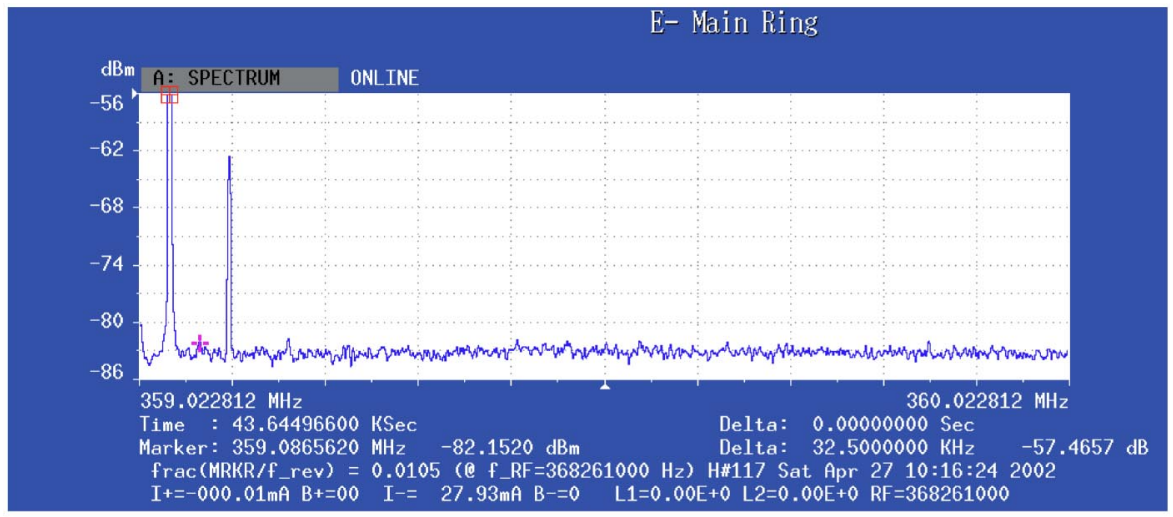

FIG. 15. (Color) Electron single bunch frequency power spectrum shows $q$-pole excitation at $26 \mathrm{~mA}$.

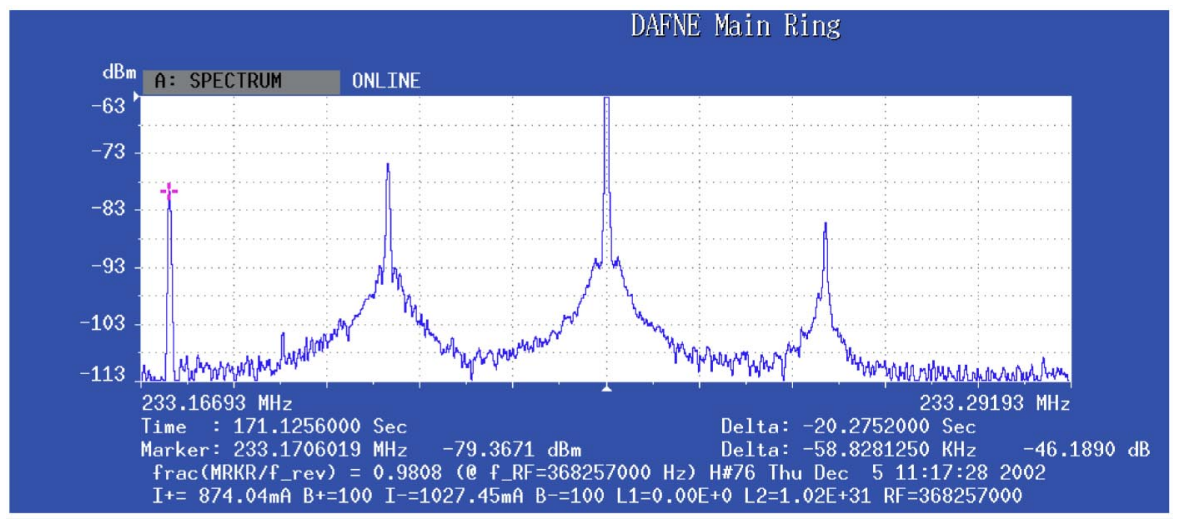

FIG. 16. (Color) Positron beam frequency power spectrum showing a $q$-pole excitation at $874 \mathrm{~mA}$, in collision (the $q$-pole left sideband has the cross marker, the right sideband is missing, the revolution harmonic in this case is in the middle).

to control the dipole motion without interfering with the quadrupole one. The $q$-pole instability threshold has again limited the total beam current.

This fact has been interpreted as a definitive proof that the longitudinal quadrupole instability is not generated
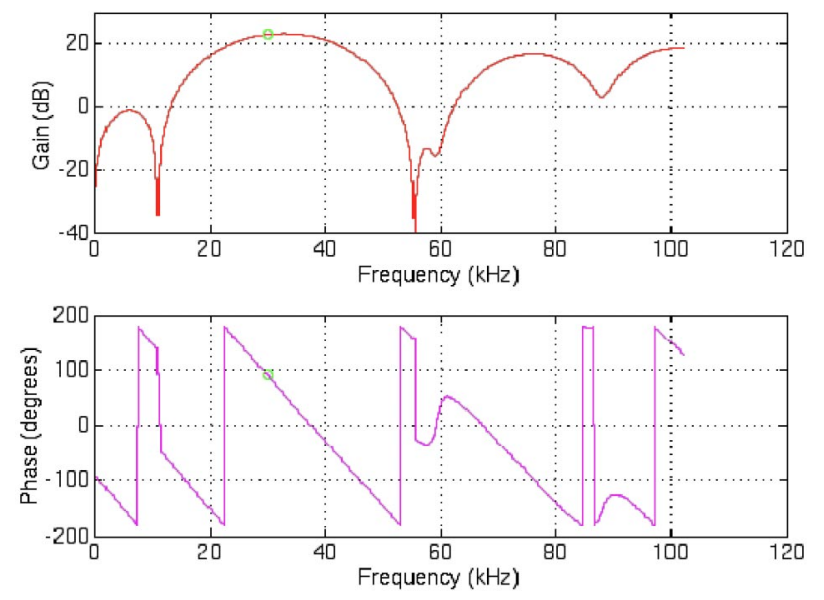

FIG. 17. (Color) Amplitude and phase response versus frequency response of a FIR filter with a peak at $30 \mathrm{kHz}$ and a notch at $56 \mathrm{kHz}$. by the LFB. The instability is self-excited, i.e., the LFB itself does not create it. In our opinion, the machine impedance is a possible source of the instability. This agrees also with early theoretical prediction of the quadrupole instability in DA $\Phi$ NE, based on the collider broadband impedance estimates [18]. A double water bag distribution model and numerical simulations have been used to solve the Vlasov equation in order to investigate the bunch longitudinal coherent mode coupling leading to the microwave instability and to evaluate the instability thresholds. In certain machine conditions, the quadrupole mode threshold has been calculated lower than dipole and sextupole ones: $23 \mathrm{~mA}$ of bunch current with $V_{\mathrm{rf}}=100 \mathrm{kV}$. Such mode coupling has been foreseen leading to bunch shape modulations potentially harmful for beam-beam interactions.

In the base at the interpretation of the phenomenon, the frequency shift described in Sec. III A should be due to a partially incorrect phase response of the LFB at the quadrupole frequency.

A third test has been done: changing the front-end LFB signal sign (see Fig. 14) and kicking the bunch on the tail (instead of the head), we succeeded in the control of the beam. This is an experimental proof that kicking the 


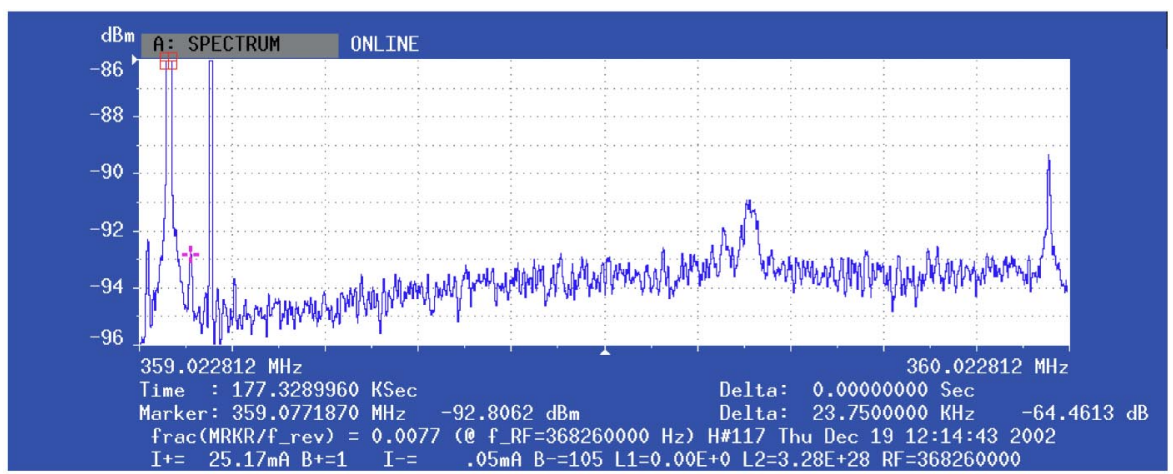

FIG. 18. (Color) Positron single bunch frequency power spectrum shows a $q$-pole excitation if $I_{b}>23 \mathrm{~mA}$ and LFB off. The revolution harmonic is with the square marker, the dipole oscillation with the cross marker, and at its right the $q$-pole oscillation.

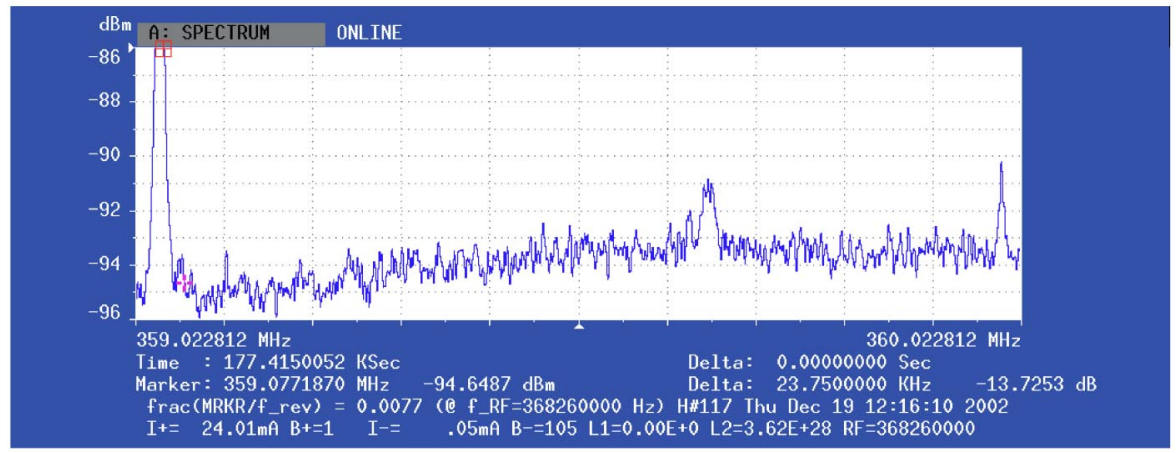

FIG. 19. (Color) Same configuration of the previous figure, but with the LFB on and correctly set: the $q$-pole excitation is damped.

bunch head or the bunch tail is equivalent to an inversion of the phase response sign of the feedback for the $q$-pole motion, i.e., the $q$-pole feedback phase response maintains the same sign after the double inversion.

The last experiment that we have done (in December 2002) is documented by the following two figures. In the positron ring, a single bunch shows self-excited quadrupole oscillations with current $>23 \mathrm{~mA}$ and LFB off; see Fig. 18. By turning on the LFB and setting it correctly, it has been possible to easily damp the quadrupole motion as shown in Fig. 19. This confirms again that the LFB system, designed to damp synchrotron oscillations, is able to damp the longitudinal quadrupole ones not only in multibunch but also in single bunch and in both rings.

\section{CONCLUSIONS}

The more relevant conclusions are two: (1) The quadrupole instability is self-excited and not caused by the LFB system. (2) The broadband time-domain feedback system is able, if correctly set in all its parts, to damp effectively the quadrupole instability together with the dipole one. This is possible if the following conditions are observed:

(i) The $q$-pole oscillations must be detected in the front end with same sign phase offset for all bunches; this depends on the LFB architecture (implementing individ- ual but identical filters for each bunch) and by considering that the FE signal can be described [19] by

$$
x_{n}(t)=i_{b}\left(1+a_{q} \sin \omega_{q} t\right)\left(\phi_{\mathrm{dc}}+a_{d} \sin \omega_{d} t\right)
$$

where $i_{b}$ is the bunch current, $a_{q}$ and $\omega_{q}$ are the $q$-pole amplitude and frequency, $\phi_{\mathrm{dc}}$ is the FE phase offset, and $a_{d}$ and $\omega_{d}$ are the dipole amplitude and frequency. From Eq. (3), the sign of the detected quadrupole oscillations depends on the phase offset sign.

(ii) The $q$-pole frequency must be managed inside the LFB loop together with the dipole one, providing the correct damping response in gain and phase for both signals, dipole and quadrupole; i.e., the global phase response of the feedback must give a $\pi / 2$ phase shift at both frequencies.

(iii) It is necessary to apply a different kick to the bunch head and to the bunch tail: this is because the $q$-pole motion is not a rigid longitudinal oscillation of the bunch, but it is an intrabunch longitudinal oscillation. This confirms that a two-macroparticle model (head and tail) $[20,21]$ is convenient for explaining the mechanism. If the bunch length is too small with respect to the distance peak to zero of the BE periodicity, the feedback kick could be ineffective to damp quadrupole and dipole motion together. 


\section{ACKNOWLEDGMENTS}

Thanks to A. Hoffman for an interesting even though informal meeting held in December 2001 at SLAC. Shyam Prabhakar has suggested how to modify the bunch pattern and has kindly provided a software tool to calculate the effects on the $q$-pole threshold. Thanks to Mario Serio for many discussions.

[1] M. Zobov et al., in Proceedings of the EPAC 2002, Paris, France (EPS-IGA/CERN, Geneva, 2002).

[2] L. Sapozhnikov et al., in Proceedings of the Beam Instrumentation Workshop, Santa Fe, New Mexico, 1993 (SLAC Report No. SLAC-PUB-6365, 1993).

[3] G. Oxoby et al., in Proceedings of the 4th European Particle Accelerator Conference (EPAC 94), London, England, 1994 (SLAC Report No. SLAC-PUB-6520, LBL-35790, 1994).

[4] J. D. Fox et al., in Proceedings of the 4th European Particle Accelerator Conference (EPAC 94), London, England, 1994 (SLAC Report No. SLAC-PUB-6548, LBL-35913, 1994).

[5] D. Teytelman et al., in Proceedings of the Sixth Beam Instrumentation Workshop (BIW94), Vancouver, 1994 (SLAC Report No. SLAC-PUB-6675, LBL-36174, 1994).

[6] S. Bartalucci et al., Part. Accel. 48, 213-237 (1995).

[7] D. Teytelman et al., in Proceedings of the 16th IEEE Particle Accelerator Conference (PAC95), Dallas, Texas, 1995 (SLAC Report No. SLAC-PUB-95-6879, LBL37210, 1995).

[8] D. Teytelman et al., in Proceedings of the 7th International Conference on Accelerator and Large
Experimental Physics Control Systems, 1999 (Comitato Conferenze Elettra, Trieste, Italy, 1999), pp. $252-254$.

[9] J. D. Fox et al., in Proceedings of the 9th Beam Instrumentation Workshop (BIW 2000), Cambridge, MA, 2000 (SLAC Report No. SLAC-PUB-8410, 2000), pp. 226-233.

[10] S. Prabhakar, Ph.D. thesis, Stanford University, 2000 (SLAC Report No. 554, 2000).

[11] H. Hindi et al., "PEP II: RF and Feedback R\&D," Particle and Fields'92, Batavia, IL, 1992 (SLAC Report No. SLAC-PUB-5979, 1992).

[12] D. Teytelman et al., in Proceedings of the 9th Beam Instrumentation Workshop (BIW 2000), Cambridge, MA, 2000 (Ref. [9]), pp. 218-225.

[13] R. Boni et al., Part. Accel. 52, 95-113 (1996).

[14] R. Boni et al., in Proceedings of the 5th European Particle Accelerator Conference (EPAC 96), Sitges, 1996 (Report No. LNF-96/033(P), 1996.

[15] A. Ghigo et al., in Proceedings of the EPAC 2002, Paris, France (Ref. [1]).

[16] A. Drago et al., in Proceedings of the EPAC 2002, Paris, France (Ref. [1]).

[17] E. Kikutani, Part. Accel. 52 251-273 (1996).

[18] M. Zobov et al., DAФNE Technical Note No. BM-3, 1998.

[19] A. Drago et al., DAФNE Technical Note No. BM-10, 2002.

[20] A.W. Chao, Physics of Collective Beam Instabilities in High Energy Accelerators (John Wiley \& Sons, Inc., New York, 1993, cited in [17].

[21] A.W. Chao and M. Tigner, Handbook of Accelerator Physics and Engineering (World Scientific, Singapore, 1999), p. 104. 